\title{
Practical approach to knowledge management implementation in historic buildings restoration
}

\author{
Katayoun Taghizadeh ${ }^{\mathrm{a}}$, Eskandar Mokhtari ${ }^{\mathrm{b}}$ and M.H. Mahmoudi Sari ${ }^{* *}$
}

${ }^{a}$ Associate Prof., Department of Architecture, University of Tehran, Tehran, 1417466191, Iran

${ }^{b}$ Assistant Prof. and Project Manager, Research Institute of Cultural Heritage and Tourism, Tehran, Iran

${ }^{c}$ PhD Candidate in Project Management and Construction, Department of Architecture, University of Tehran, Tehran, 1417466191, Iran

\section{H R O N I C L E A B S T R A C T}

Article history:

Received June 4, 2014

Accepted 29 October 2014

Available online

November 12014

Historic building

Restoration

Knowledge Management (KM)

Conceptual model

KM inner and outer cycles

\begin{abstract}
The aim of this investigation is to establish an applicable model for knowledge management implementation that works suitably in organizations involved in projects defined for a historic building such as restoration. Based on a peer review on general approaches in literature, some main improvement potentials are recognized to enable the model to meet with the especial objectives, which shall be considered in such projects. Content analysis was applied to ascertain the new presented model capabilities and improvement in each of three main core components of knowledge management implementation model including peoples, processes, and technologies. We came up with a new conceptual model named as Finger Frame Model with four individual tiers connected with an intelligent communication medium .

It is concluded that three main components of KM block diagrams consist of knowledge identification, acquisition, and prevention are appropriately covered in basic tier. The other three components of inner cycle of KM basic diagram consist of using, development, and distribution are also covered in an interface between operators and basic tiers, which together with make lower frame of new presented model. The model is in a continuous improving stage considering self-monitoring processes in an individual tier with the same name, which is located in the upper frame. An upper layer named as goal tier is also located in top frame in which, the main quantitative goals of $\mathrm{KM}$ implementation in the project organization are considered. The conceptual diagrams as well as internal processes of each main tier are also presented and discussed. Based on the overall explanations, some main benefits and risks are highlighted for new presented model and outlooks are debated. The model is applied in a real case study and the results are compared with the previous knowledge management implementation model. The results show that the model works efficiently in projects organization related to the historic building restoration.
\end{abstract}

\section{Introduction}

Historic Buildings (HBs) shall be considered as major effective parameter that influences tourism industry. Managing and restoration of HBs is a developing market that its effects on this industry as well as economic behavior of countries heritage cannot be ignored. Countries with a lot of cultural resources, such as Iran, have an important economic opportunity in this international market. One of

*Corresponding author.

E-mail addresses: mahmoudisari@ut.ac.ir (M.H. Mahmoudi Sari) 
the main issues about optimized managing of preservation, reconstruction, and restoration of HBs, is to reserve any technical and scientific knowledge, which can be applied in such projects. Knowledge management $(\mathrm{KM})$ in any implementation models that presented up to now can be assumed as a reliable method to reserve and to take into account any specific consideration that shall not be neglected for each HBs related project. However, as per recent researches, special approaches are innovated to optimize knowledge management models including site specific variables in a country with ancient civilization.

Some main reasons can be interpreted for preference of site-specific knowledge management models rather than general models that developed without considering special issues about HBs-related projects. Special conditions of these projects due to the applicable materials and methods can be explained as a main reason. Local technologies and materials are mainly applied in restoration of HBs, which is highly affiliated to the local sciences and experiences. Identification, acquisition, and use of this knowledge is principally related to the site-specific methods that can be embraced in an applicable model which is developed considering special condition that govern the HBs-related projects.

In this work, based on a peer review on general approaches to knowledge management implementation, an applicable model is presented and suggested to implement knowledge management especially in restoration of HBs. The main goal of the new presented model is to reduce the bottlenecks in identification, acquisition, and use of necessary knowledge for restoration of a historic building. The method applied here is to minimize the role of personalize sciences and restoration methods in the project and to replace it with standard methods that established based on all technical and experimental which can be functional in HBs restoration.

\section{Evaluation of Approaches to KM Implementation}

As a basis of all specific approaches to manage any sort of knowledge in HBs related projects, the KM implementation models, which were developed in a general manner shall be assessed. It is posited that knowledge identification, acquisition, and application proceed in well-defined routes only if the main definitions of KM customized for a specific goal. In addition, any new customized approach can be followed reliably if two main elemental phrases of KM are completely satisfied. Firstly, eight main components of building blocks of KM, i.e. knowledge identification, acquisition, preservation, distribution, development and use shall be covered. This covering shall be taken into account in two main rotations in the project including inner and outer cycles. Secondly, aligning three core components of KM consist of peoples, processes, and technologies. Therefore, the assessment of the KM implementation models, which were presented in the literature in a general approach is done here considering these two main milestones. The main goal of evaluation is to recognize the points that may be applied in focusing during design of new KM model to improve quality of outputs by safeguarding and enhancing of skills, capabilities, experiences, routines, norms, and technologies in HBs restoration.

According to the authors' reviews, pioneer leading to the KM modeling can be found in Bowers (1970). In this general model the identification, prevention and acquisition of knowledge are rationally covered. However, the distribution techniques are limited and also there is not an integrated system for transferring and development of sciences. The normative goals in this model is mainly defined by plus worth generation caused by model implementation. Related processes were also presented based on the defined goal. Similar approaches can be seen in next model developer such as Barely (1986) with more emphasize on the peoples role in the implementation model. Hambrick (1994) tetragonal model added a new individual layer to his proposed model for definition of the goals and the operational as well as strategic knowledge goals were mainly considered in the model. Chesbrough (1999) came up with a same manner but with obvious increasing in the strength of 
implementation model processes. Recording, transferring, and simultaneously evaluation of knowledge were added by Bhatt and Ganesh (2000) to transfer the personal to organizational knowledge. They also predicted some complementary processes to compile the implicit knowledge to change it to explicit forms. Considering the direct pointing to knowledge management implementation model, the first approaches can be found in Nonaka (2001) works. He and his coworkers suggested clear routines and processes for better and faster sharing and exploiting knowledge and technologies to have a higher performance in the project organization. Effective informal networks were applied to develop the implementation model of project knowledge by Ferraro et al., (2005).

To minimize the uninformed decisions and to achieve sufficient transparency in internal and external knowledge in the project organization, they defined an effective informal network as an upgraded process in their implementation model. The appropriate building levels for various capability of the project organization are also defined in the network. Alignment of three core components of KM is suitably covered in their enterprise model, but there is not any measurement or monitoring tier in the model. The insufficiency of individual tier for goals in model was approximately reimbursed by Hoffman (2005) and the other general path was covered by him. Knowledge-based goals are proposed as a fast competition way for project organization. Also considering various operators in the definition of model goals such as stakeholders as well as sponsors are discussed and the effects of this approach to goal definition on the outputs of implementation model were evaluated. Similar implementation approach was developed by Lustri (2007) with more emphasizing on application (use), distribution, and development of knowledge. Relevant processes were applied to integrate the knowledge in any form throughout the organization and to minimize the role of persons in the technical outputs of the design or construction procedures and specifications. Bade (2011) applied these improvement in a new communication media which can be assumed an added technology especially applicable for integrating any data transferring based on the specified protocols and evidences which can be clarified and verified in project organization. In the other word, he developed technology as one of the main core components of KM implementation model.

Parallel to the general approaches to knowledge management implementation that described before, some of recent researchers developed some models to be applicable for management of cultural heritages. Although the KM is not especially covered in their works but for covering any related investigations and innovation about the topic of this research, it is assessed considering the main issues in KM in cultural heritage related projects. Chutinan (2008) developed an integrated plan with a secondary general part for KM to optimize the structure of the organization which is responsible for effective promotion of heritage property and applied it for a historic park in Thailand. A similar approach was also developed by Tarhuni (2012) for integrating management especially for HBs in Libya. In these works, main components of KM are generally covered as a subordinate model and the main achievement of previous KM implementation model are applied and some main contribution about integrating management of cultural heritage areas and HBs management were presented that are not in the main scope of our work.

Combining the assessment results on each KM implementation modeling approach which mentioned above, leads to some clear axes to be focused for improvement. Enhancing the capabilities of model shall be categorized based on three core components of KM implementation modeling, i.e. peoples, processes, and technologies. The details of improving procedure as well as predicted combined model are discussed in next session.

\section{Practical Approach to KM in HBs-Related Projects}

From the fact and figures, it shows that any improvement in KM implementation models shall satisfy clear objectives in core components of KM. The authors are in the view that although the previous 
models have a lot of objects to follow in any project organization such as HBs-related projects, they do not induce the project teams to observe special characteristics of a HB restoration project. From the investigation of the pioneer approaches to KM implementation model and considering essential requirements in a HBs-related project such as restoration, the main axes that are recognized for improving in a new KM implementation model is listed in Table 1.

The main improvement potentials shall be considered in a combined conceptual model for KM implementation. The arrangement of the tiers in new combined model is instructed based on the applicable approaches in pioneer works which were inspected in previous session and new ideas for better working of the model is implemented to create a new arrangement. As it is illustrated in Figure 1, the proposed KM implementation model included four main tier consist of Basic Tier, Operators Tier, Monitoring Tier, and Goals Tier. Relation between these main tiers is established by an intelligent interconnecting loop. Roles and position of operators are considered in an individual tier named as operator tier. Requirements or dependencies of each operator are completely related to its role (horizontal coordinate) and position (vertical coordinate). The requirements can be evaluated as critical or open based on the coordinates of the operators. The key idea is to focus on the coordinated of operators I all model activities. Action framework of the presented model is dependent to knowhow techniques which shall be applied for fulfillment of quantitative goals which are located in an individual header tier named as Goal Tier.

Relation between goals and tasks are considered in an intelligent communication medium which is the transferring, calling, checking, and recommending media works together with four main tiers of the model. The monitoring tier is responsible for evaluation of the model operation which is utilized within the quantitative procedures act on some main inputs received from the model via intelligent communication medium. The intelligence behavior means that the automated communication medium is able to detect and trace malfunctions or any unusual operating by comparing and referring to the databases implemented in basic tier. Moreover, a list of links to the appropriate technical data, according to the project stage such as design, material selection, construction or maintenance of HBs restoration, will be provided for corresponding operator.

\section{Table 1}

Main improving potentials to be considered in a new applicable KM implementation model for HBsrelated projects

\begin{tabular}{|c|c|c|}
\hline No. & $\begin{array}{c}\text { KM } \\
\text { Core Component }\end{array}$ & Improving potentials in a new KM implementation model \\
\hline 1 & \multirow{3}{*}{ People } & $\begin{array}{l}\text { Dividing peoples in parallel sessions based on their role or type of influence on } \\
\text { HBs related project; definition of horizontal coordinate }\end{array}$ \\
\hline 2 & & $\begin{array}{l}\text { Dividing peoples in several groups based on their position or level of influence on } \\
\text { HBs related project; definition of vertical coordinate }\end{array}$ \\
\hline 3 & & $\begin{array}{l}\text { Definition of an individual tier in model especially for peoples including all } \\
\text { operators in various hor. and ver. Coordinates }\end{array}$ \\
\hline 4 & \multirow{3}{*}{ Process } & $\begin{array}{l}\text { Definition of a new process in model for calculating of worth plus caused by KM } \\
\text { implementation especially in a HBs-related project }\end{array}$ \\
\hline 5 & & $\begin{array}{l}\text { Adding a new individual tier to the model for implementation of self-evaluation } \\
\text { processes and feeding back to other tiers }\end{array}$ \\
\hline 6 & & $\begin{array}{l}\text { Making intelligent as a new capability in all model processes consist of analysis } \\
\text { and communication processes }\end{array}$ \\
\hline 7 & \multirow{3}{*}{ Technology } & $\begin{array}{l}\text { Integrating various tiers in a coverage applicable model for together working by } \\
\text { entering the implementation technology in all tiers }\end{array}$ \\
\hline 8 & & $\begin{array}{l}\text { Definition of special characterizes for applying in HBs related projects to increase } \\
\text { the applicability of model }\end{array}$ \\
\hline 9 & & $\begin{array}{l}\text { Definition of technological transferring protocols to apply in an integrated } \\
\text { software }\end{array}$ \\
\hline
\end{tabular}


The actions for fostering the supportive processes in intelligent communication medium are monitored by an individual element of the model located in monitoring tier. The result of verification collaborated based on monitoring and checking procedures run in this tier. It causes reduction of the time needed to make the model operation reliable especially in HBs-related projects. The validation of usability of the proposed conceptual model is also done in a higher level by top layer acts as Goal Tier. Decomposition of the goals in this layer is considered. Decomposed data received from other tiers use to evaluate the fulfillment of quantitative goals.

The domain of new KM implementation model presented here is especially the projects related to HBs, so the processes and methods used in the piloting of the model in all four layers are defined according to the main issues of this domain. This definition is made using appropriate characteristics in related check lists and matrixes as a part of model data base which is located in all tiers. All of the related rules for requests analysis and measurement planning are carried out based on the defined characteristics.

The applied characterizing methods can be updated after piloting of the presented model and feedback evaluation comes from both monitoring and goal tier. To be able to follow the two inner and outer cycles of KM flow diagram, it is important to divide conceptually the four main layers or tiers of the presented model based on the eight blocks of the diagram. The processes and data transferring channels are developed based on this separation in the model.

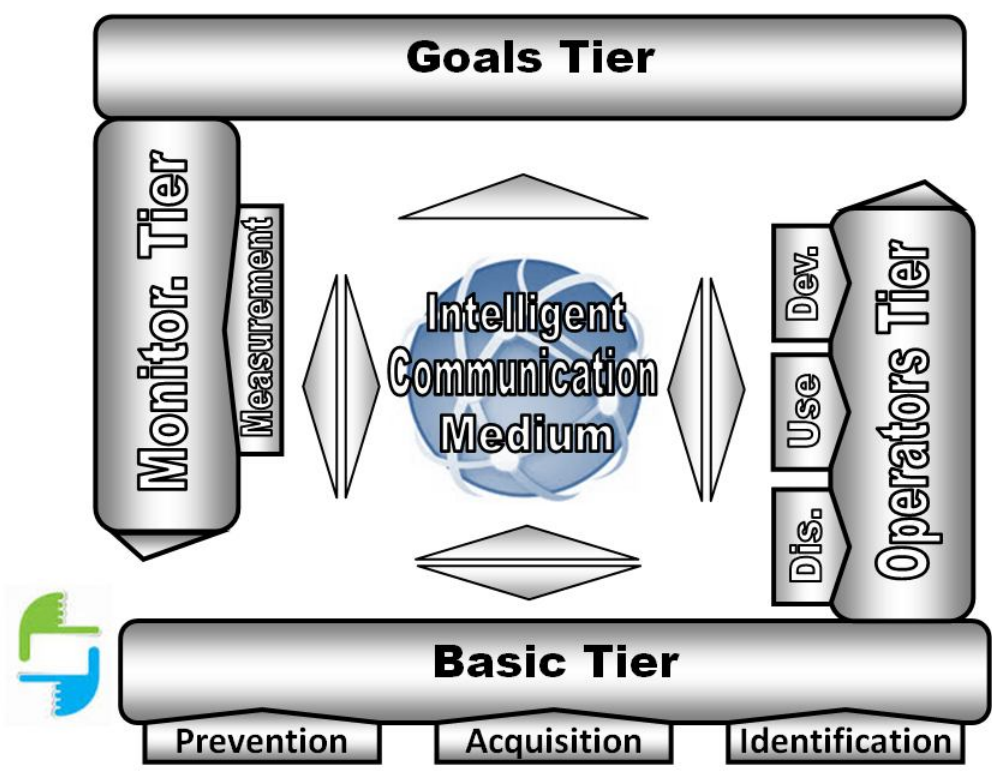

Fig. 1. New conceptual model for KM implementation in HBs-related project organizations, hereafter named as Finger Frame Model (FFM)

This dividing leads to an applicable arrangement in horizontal and vertical communicating methods which are defined in the medium. High impact on the results of monitoring and evaluation can be expected from this arrangement. Especially for HBs-related projects such as restoration, recordable and routine communicating methods are not only the transferring ways of the knowledge. Many personalize conduction such as reliable empirical methods in material selection and mixing, construction and even management need to be taken into account. These occasions have relatively more or less impacts on characterizing procedure and definition of technical processes in the proposed model. 
Determination of probable faults, preparation of fitting modification offer, appraising caused damages and related side effects, and proposing alternatives to self-correction of the model can be explained as a part of monitoring tier in the proposed model. Settlement of abnormal events is also expected from this individual tier. Extensibility of the model is a capability of all tiers. In basic layer, where technical databases, national and international rules, parameterized essential data for any analysis processes of the model are located, the extensibility is possible by developing and updating all of mentioned items. It is also expected for monitoring tier by developing the monitoring procedures located in it. The extensibility in goal tier is expected by increasing the main checking procedures located in this layer to evaluate the achievement of quantitative goals. The operators tier is also extensible because of the potential increasing in parallel sessions of operators or in more detail dividing of them based on the role and position of them in $\mathrm{Km}$ implementation system.

Any cultural-based routes for implementing KM in the project organization are also covered in the presented model. Necessary infrastructure for these routines such as training and instructing procedures for any operator or peoples evolving in a HBs-related project are considered in four main tier as well as communication medium. The organized knowledge which generated or gathered in the project is productively deployed in the project and saved for other similar or related project as one of the KM implementation model. The first concern in explanation of finger frame model is the reason for separating the tiers into the two main frames; upper and lower. As it is clearly indicated on Figure 1 , all of the six elements of the inner cycle of KM block diagram are covered in the lower frame. In contrast, goals setting as well as knowledge measurements as two main elements which added in outer cycle of KM block diagram are covered in upper frame.

Locating each of the elements in both inner and outer cycles of KM diagram in lower and upper frames are completely dependent to the task and position which are defined for each tier in our proposed model. Basic tier has a main role and have been mainly affected via three main components of inner cycle, i.e. identification, acquisition, and prevention of understandings in the HBs related project. Similarly the acts and processes related to distribution (Dis. in Fig.1), use, and development (Dev. in Fig.1) are mainly covered according to the tasks and roles which are defined for operators tier. So, rationally these two tiers, i.e. basic and operators are classified in a same category. Surely all of the missions of these two layers can only be carried out simultaneously by working with upper frame tiers.

This co-working is performed via a smart interface named as intelligent communication medium. Enterprise software will be applied as this smart medium that will be discussed in the next paper. Categorizing of two tiers in the upper frame in the new model proposed here is also based on the behavior expected from these layers and relation between this behavior and two outer blocks in KM fellow diagram. So, two upper and lower frames like a frame makes by each hand are rationally created and leads to a likely name for our presented model hereafter named as Finger Frame Model (FFM).

As it can be seen in Figure 1, two-way transferring mechanisms are considered for relation between all tiers in the model via central communication loop. It means that all main mechanisms govern on the model such as calling from basic layer, acting instructions by operators with various horizontal and vertical coordinates, and monitoring the processes and communications, are completely carried out with both sending and receiving data between various tiers. The only one-way transferring data is predicted for top layer named as goal tier because of the pure receiving behavior of this layer. The results of data processing in this layer which done for quantitative evaluation of fulfillment of goals will not feed back to the model layers and will be applied to general evaluation and verification of the model concept. 


\section{Design of Operational Processes in FFM Tiers}

Following the structure presented in Figure 1, the details of operational processes which are defined for each main tier in our new presented KM implementation model (Finger Frame Model; FFM) can be designed. The processes are defined to provide reliable evidence for supporting the improvement claim which was explained in previous sessions. The operational processes are instantiated for applying the model in HBs-related projects and especially for restoration circumstances. In any other conditions with different influence parameters, the model are held in more general pattern and it shall be detail designed again considering new condition.

Before interfaces in the model can be defined or designed, the requirements, roles, position and tasks which are expected from each individual tier in the model have to be identified. When starting a new HBs-related project such as restoration, a simple question must be replied: whether existing technical knowledge as well as related experiences can help in performing it. Consequently, the project organization shall be utilized with any understanding embedded in the organization in a categorized, classified, and hierarchies groups. This first step begins with the first fundamental layer which is named in FFM as Basic Tier. Details of operational processes and acting elements in basic tier are illustrated in Figure 2.

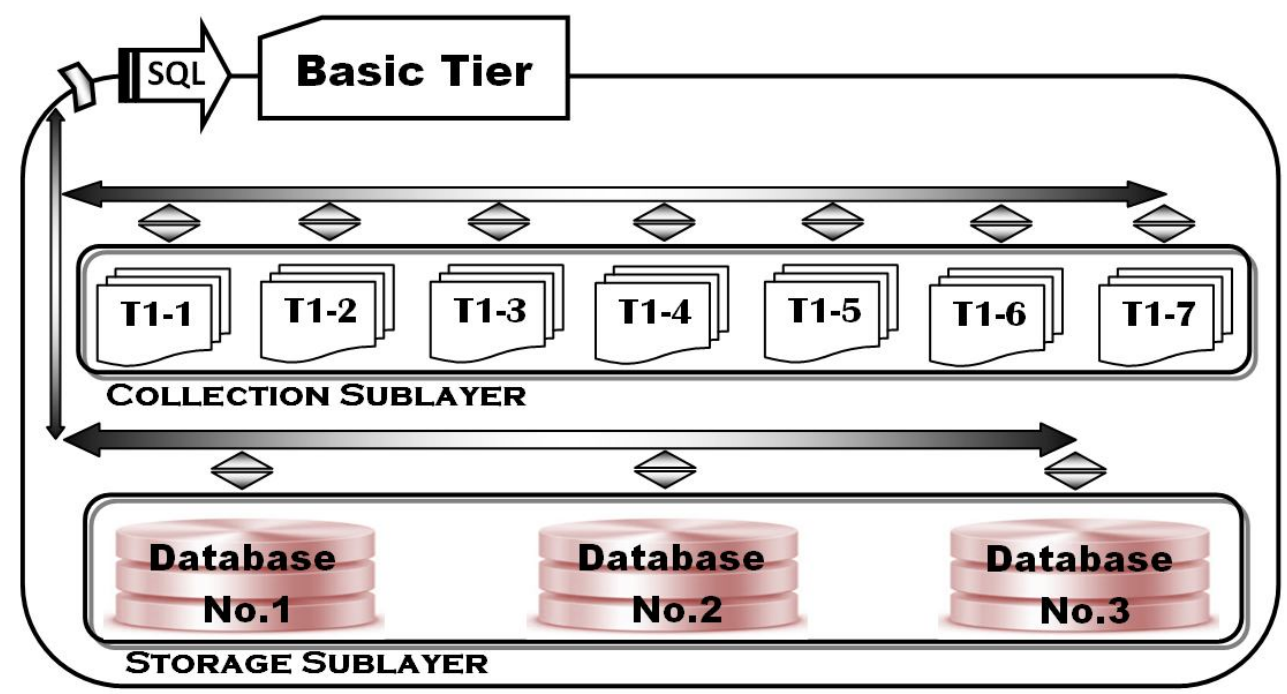

Fig. 2. Details of operational processes and acting elements in Basic Tier of FFM

As it can be seen in Fig. 2, two main sub-layers are included in the basic tier of FFM; knowledge storage and knowledge collection sub-layers. In storage sub-layer, three major databases are located. The main database consists of identified, codified and classified store knowledge about main specifications of registered and non-registered national historic buildings. The packaged knowledge about any ancient or new method or material which can be applied in a HBs-Related project is included in the second database. The third database is storage of knowledge which is identified by "lessons learned" protocols in any previously-performed HBs-related project. The two-way data transferring processes which are indicated in Fig. 2 mean that using as well as updating are simultaneously predicted in any communication with this model sub-layer. An electronically evaluation system is considered to evaluate any new identified knowledge in storage sub-layer. Evaluation processes are also utilized this layer to determine whether the knowledge is valuable to repository.

In the second sub-layer of basic tier, seven main document collections are located. Numbering procedure of these knowledge collections are based on their priority as well as relating to the first model tier (T1-i). The classified collected data about materials and technical methods for historic 
building restoration which is selected for a specific HBs-related project is gathered in the first collection. In the second collection, all of the national rules, regulations, laws, and practices related to any action on historic buildings are electronically stored. Similar documents which are established in international level are included in the third collection. Any information which is collected during special field operations for a specific historic building is gathered in the fourth collection. Sitespecific field observations and measurements such as surveying, soil mechanic investigations, damage inspection and monitoring, settlement control, and any similar data are collected here. Some of historic buildings as cultural national heritages have special social issues. These issues and concerns are classified and stored in fifth collection. Public users and nongovernmental organizations which are active in cultural heritage domain may have some issues that affect the HBs-related projects or priorities. This information is collected in the sixth collection. The last one is belong to data and documents related to the special considerations which have been followed within the client of a special HBs-related project. An internal electronically based system using SQL is applied for any relation between internal sub-layer elements as well as external calling or transferring data in basic tier. As it was explained, the major part of identification, acquisition, and prevention of HBs-related knowledge has been carried out by the main sub-layers and protocols in basic tier. Table 2 introduces the main terms indicated in Fig. 2.

Table 2

Description of the terms indicated in Basic Tier block diagram (Fig. 2)

\begin{tabular}{lll}
\hline No. & \multicolumn{2}{c}{ Basic Tier } \\
Component
\end{tabular}

Alignment between all users, actors, and operators which influence the implementation model is considered in an individual tier named as Operators Tier. As it was abstractly mentioned in previous session, all peoples that may have effects on a HBs-related project knowledge circuit are gathered and collected in this tier. Direct connection between this tier and basic tier in the model is predicted in lower frame of FFM because of the covering on three components of inner cycle of KM block diagram, i.e. users, knowledge distribution, and development.

These blocks are considered via interconnection protocols between basic and operator tiers. User's interfaces and acting is done in model via intelligent communication medium. In special application area defined in this manuscript, the all operators which may have effects on the knowledge implementation model can be categorized in seven horizontal groups and simultaneously in three vertical classes. The horizontal groups are defined as several roles of operators or horizontal coordinates here. Seven various roles consist of sponsors, stakeholders, executive directors, consultants, designers, contractors, and vendors. Three various positions which can be defined in each role consist of end user, specialist, and supervisor. Location of seven roles as well as three positions in operator tier is shown in Figure 3. The definition of each position of peoples in operator tier as well as in all $\mathrm{KM}$ implementation model can be categorized as follow. 


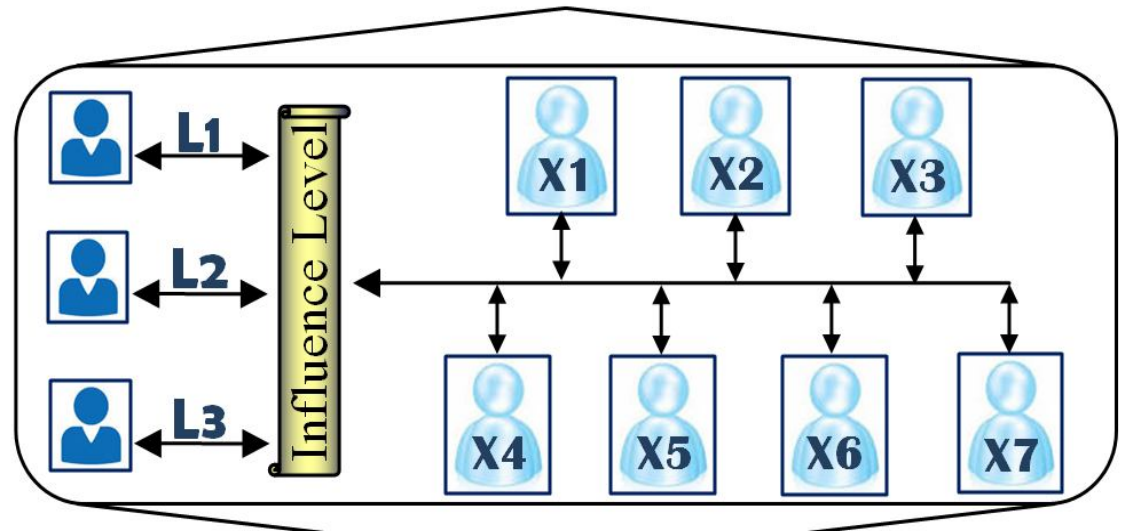

Fig. 3. Details of functional processes in operator tier of FFM considering roles $(\mathrm{Xi})$ and position $(\mathrm{Li})$ of operators involved in a HBs-related project

End user is one who only demands a special part of organizational knowledge to apply it in its special task according to its role in the project organization. His or her required knowledge is automatically provided for him or her immediately after definition of demand according to the role of him and the essentials of HBs-related project. End users are not allowed to any change, modification, updating, and even customization of knowledge in the model. The only effect from end users on the model is demand definition. Immediately after demand definition, end user will be utilized by the required knowledge as well as procedures for acting the expected tasks via other parts of the model.

Specialist is one who can apply the knowledge as an end user, but moreover, he can implement any changing, correction, updating and voiding in basic tier of the model. Both sub-layers of basic tier can be affected by operators with expert position in operator tier. The instructions for any changes are completely considered in communication protocols of the model. The causes and effects of any changes are traceable for future monitoring and inspection by higher level operators. As it was mentioned, an expert position covers all authorities of an end user. Note that any position can be defined in any role of operators because of the various horizontal coordinates of operators can be corresponding to any vertical coordinate.

Table 3

Description of the terms indicated in Operators Tier block diagram (Fig. 3)

\begin{tabular}{cclccl}
\hline No. & Operators Tier & \multicolumn{1}{c}{ Description } & No. & Operators Tier & \multicolumn{1}{c}{ Description } \\
\hline $\mathbf{1}$ & $\mathbf{X 1}$ & Sponsors & $\mathbf{6}$ & $\mathbf{X 6}$ & Contractors \\
$\mathbf{2}$ & $\mathbf{X 2}$ & Stakeholders & $\mathbf{7}$ & $\mathbf{X 7}$ & Vendors \\
$\mathbf{3}$ & $\mathbf{X 3}$ & Executive Directors & $\mathbf{8}$ & $\mathbf{L 1}$ & End user \\
$\mathbf{4}$ & $\mathbf{X 4}$ & Consultants & $\mathbf{9}$ & $\mathbf{L 2}$ & Specialist \\
$\mathbf{5}$ & $\mathbf{X 5}$ & Designers & $\mathbf{1 0}$ & $\mathbf{L 3}$ & Supervisor \\
\hline
\end{tabular}

Supervisor is one who has to monitor all ordinary and abnormal behaviors of knowledge transferring circuit in the project, any effects caused by experts, and any demand defined by users as well as any knowledge and instructions provided for him or her for doing the task. In the other word, supervisor is authorized for using of knowledge (as end users), changing in basic tier elements (as experts), and monitoring the procedure of working lower levels of operators as well as checking the results of KM implementation models based on checking the fulfillment of strategic and operational goals of the model. The main terms indicated in this figure is listed in Table 3. Supervisors can raise any need to change, improvement, or case-specific modification in transferring protocols, instructions, tasks, and communication for better and faster working of the model. As it is illustrated in Fig. 3, seven roles of operators acting on project organization are located on horizontal axis. Each of these roles can have 
three alternatives based on their influence level on KM implementation model. These alternatives named as vertical positions of peoples in operator tier of FFM.

\section{Discussion of the Presented Model}

Based on the details presented before for new suggested model, its main benefits and risks can be discussed. The model is developed to be applicable in area of concern of this manuscript, i.e. HBsrelated project organization. The intended benefits of the new model named as FFM can be described especially considering the requirements of knowledge management in area of concern. Possible risks can also be determined based on the initial evaluation of the model.

The new model has been created with emphasizing on dividing any knowledge-based processes including operational processes and coordination processes according to roles and positions of the operators act in a project organization. The model has been developed with providing assistance in the suit planning and execution of the KM main core blocks. Performing the presented model and verification of it in a case study is done and the results are under preparation to be published in an individual manuscript.

Based on the main basis of developing a new KM implementation model that was described, it is supposed to supply some main clear benefits. The first benefit is customizing the model with the essential and operational requirements in area of concern of this research. This benefit is simultaneously provided in peoples, processes and technologies involved and applied in a HBsrelated project such as restoration. Both management and operational views are completely matched with the concerns in such projects.

Integration of the organizational processes applied in area of concern can be assumed as other benefit. The project-oriented view of KM implementation can be seen in presented model and leads to more appropriate results in integration of operational processes used in a restoration project. Technical knowledge identification, collection, and application with a repeatable manner are considered in this integration.

Continues improvement due to self-checking procedure which is embedded in the model is another clear benefit of FFM. The monitoring procedures are responsible for going up controlling of model operation as well as any data transferring and communication between various operators involved in the project organization. The details of expected improving in the presented model in comparison of previously developed models were explained in previous sessions. About the probable risks of the model, it shall be noted that there is not any long-term experience in using the presented model. So, the feedbacks from applying it can be assumed as the main lack of data and corresponding plausible pitfalls shall be considered by readers. A main anticipated risk is in definition of the characteristics for quantization of any qualitative processes in the project.

These processes can be assumed as the procedure of calculating the plus worth in KM implementation in a project organization which is applied in goal tier of FFM. Moreover, definition of criterions to call relevant and appropriate technical data from databases by operators in various projects and various stages of a project is another pitfall. The characterizing procedures shall be updated, modified, corrected, or adjusted based on the outputs come from long term using of the model. The correct and applicable linking between several knowledge domains which influence a HBs-related project can be considered as a possible risk in presented model. It is tried to cover appropriately the various knowledge domains in an integrated database as well as an intelligent communication medium, which is utilized with updated smart acting procedure using reach collections of technical data. But it shall be verified in a real case study and shall be continuously validated in several projects in area of concern of this manuscript. 


\section{Application of the Presented Model in a Case Study}

The presented model is applied in a case study to inspect the capabilities of the model which is embedded in the conceptual model for knowledge management implementation. The case is a consulting engineers company involved in a historic building restoration named as Ghazagh-Khaneh in Tehran, capital of Iran. The company organization was applying a knowledge management model similar to Lustri (2007). At the beginning of case study, the presented model was applied only for Ghazagh-Khaneh project and other parts of the organization were made without any change relative to Lustri (2007). So, the presented model (FFM) can be compared with the previous model (Lustri, 2007) in a reliable manner. The main characteristics which were assessed in the organization are listed in Tables 4 to 7 . In these Tables, the main items that can affect the added values captured by implementation of a knowledge management model are listed. In the other word, the main achievement or improvement in the organization which can be appeared after implementation of a knowledge management model are listed. Any item in the table is assigned with a specific weight. The more effective items have mode weight, based on the experiences of the projects related to historic building restoration projects.

\section{Table 4}

Part one of the characteristics that affect added values come from knowledge management implementation in the project organization; used in the case study

\begin{tabular}{clcc}
\hline No. & \multicolumn{1}{c}{ Description } & Weight & Category \\
\hline 1 & Increasing the number of data transferring between designers and databases & 30 & AVI-1 \\
2 & Increasing the number of updating processes in technical databases & 25 & AVI-1 \\
3 & Increasing the data-interacting within organization staff & 25 & AVI-1 \\
4 & Increasing external queries to the project organization databases & 20 & AVI-1 \\
5 & Increasing the knowledge based records in the databases & 25 & AVI-1 \\
6 & Increasing the lessons-learned items in the related technical databases & 20 & AVI-1 \\
7 & Increasing the interaction with research centers related to the restoration projects & 20 & AVI-1 \\
8 & Increasing the interaction with academic centers related to the restoration projects & 20 & AVI-1 \\
\hline
\end{tabular}

\section{Table 5}

Part two of the characteristics that affect added values come from knowledge management implementation in the project organization; used in the case study

\begin{tabular}{|c|c|c|c|}
\hline No. & Description & Weight & Category \\
\hline 1 & Decreasing the revisions required for produced documents in the organization & 20 & AVI-2 \\
\hline 2 & Decreasing time delay relative to the approved project time schedule & 25 & AVI-2 \\
\hline 3 & Decreasing the essential technical meeting in the project organization & 25 & AVI-2 \\
\hline 4 & Decreasing the essential management meeting in the project organization & 25 & AVI-2 \\
\hline 5 & Decreasing re-work man-hour in the restoration project & 30 & AVI-2 \\
\hline 6 & Decreasing the required time for workflows within the project organization & 25 & AVI-2 \\
\hline 7 & Faster synchronization of the project due to the changes from client or market & 25 & AVI-2 \\
\hline 8 & Decreasing the lead times of the materials required for historic building restoration & 25 & AVI-2 \\
\hline
\end{tabular}

\section{Table 6}

Part three of the characteristics that affect added values come from knowledge management implementation in the project organization; used in the case study

\begin{tabular}{|c|c|c|c|}
\hline No. & Description & Weight & Category \\
\hline 1 & Decreasing the Man-Hour used in a restoration project & 30 & AVI-3 \\
\hline 2 & Decreasing re-work costs in the restoration project & 25 & AVI-3 \\
\hline 3 & Decreasing of stand-by time duration in the project staff & 25 & AVI-3 \\
\hline 4 & Decreasing the cost of the papers used for printing of the project documents & 20 & AVI-3 \\
\hline 5 & Decreasing the cost of materials used in the construction of a restoration project & 25 & AVI-3 \\
\hline 6 & Decreasing the cost of procurement services needed for material supply & 25 & AVI-3 \\
\hline 7 & Decreasing the negative report received from project construction & 25 & AVI-3 \\
\hline 8 & Decreasing the dependence to non-available methods and materials & 25 & AVI-3 \\
\hline
\end{tabular}




\section{Table 7}

Part four of the characteristics that affect added values come from knowledge management implementation in the project organization; used in the case study

\begin{tabular}{|c|c|c|c|}
\hline No. & Description & Weight & Category \\
\hline 1 & Decreasing the technical comments received from client technical team & 25 & AVI-4 \\
\hline 2 & Decreasing the technical comments received from internal project manager & 30 & AVI-4 \\
\hline 3 & Decreasing the technical comments received from client project manager & 30 & AVI-4 \\
\hline 4 & Decreasing technical queries received from construction team & 25 & AVI-4 \\
\hline 5 & Increasing technical instructions in the project organization & 20 & AVI-4 \\
\hline 6 & Decreasing the non-recorded experiences related to a restoration project & 30 & AVI-4 \\
\hline 7 & Increasing the number of application for technical instructions & 30 & AVI-4 \\
\hline 8 & Improvement the project organization relative to the similar organizations & 30 & AVI-4 \\
\hline 9 & Increasing the cohesiveness of the staff to the project organization & 25 & AVI-4 \\
\hline 10 & Decreasing dependence of the project to the staff especially in technical issues & 30 & AVI-4 \\
\hline 11 & Decreasing the alarms received from the client during the restoration project & 35 & AVI-4 \\
\hline 12 & Increasing the change of sciences to the internal technical instruction & 20 & AVI-4 \\
\hline 13 & Increasing the applicability of R\&D department outputs in project organization & 20 & AVI-4 \\
\hline 14 & Increasing the published records came from technical achievement in the project & 20 & AVI-4 \\
\hline 15 & Increasing the quantity and quality of the valid certificates & 20 & AVI-4 \\
\hline 16 & Increasing the variety of the technical domains for organization activities & 25 & AVI-4 \\
\hline
\end{tabular}

As it can be seen in the above mentioned four tables, the characteristics that affect added values come from knowledge management implementation in the project organization are categorized in four separate parts:

- AVI-1: Added value index, part 1; the added value is achieved due to the easiness of document availability and knowledge transferring within the project organization after KM model implementation.

- AVI-2: Added value index, part 2; the added value is achieved due to the direct saving of time within the project organization after KM model implementation.

- AVI-3: Added value index, part 3; the added value is achieved due to the direct saving of cost within the project organization after KM model implementation.

- AVI-4: Added value index, part 4; the added value is achieved due to changing implicit to explicit knowledge as well as changing personal to organizational knowledge within the project organization after KM model implementation.

The sum of the four parts is summarized in AVI, i.e., AVI $=\Sigma$ AVI- $i$ for $i=1$ to 4 . The characteristics are measured in a 15-months time period in the project organization described before and the results are discussed comparing the presented model implementation results with the previous implementation model. The comparison is illustrated in Figures 4 to 8 . The comparison is done between the results came from presented model especially in the ghazagh-khaneh restoration project and another restoration project in a parallel project organization in the same company which follow the previous knowledge management implementation model which was based on Lustri (2007) recommendation.

The results can be investigated based on the cost and time points of view and the strength and weakness of the presented model as well as the project organization reaction to the new implementation model can be assessed. Based on the assessment, the main conclusions can be achieved as it is described in the next session. 


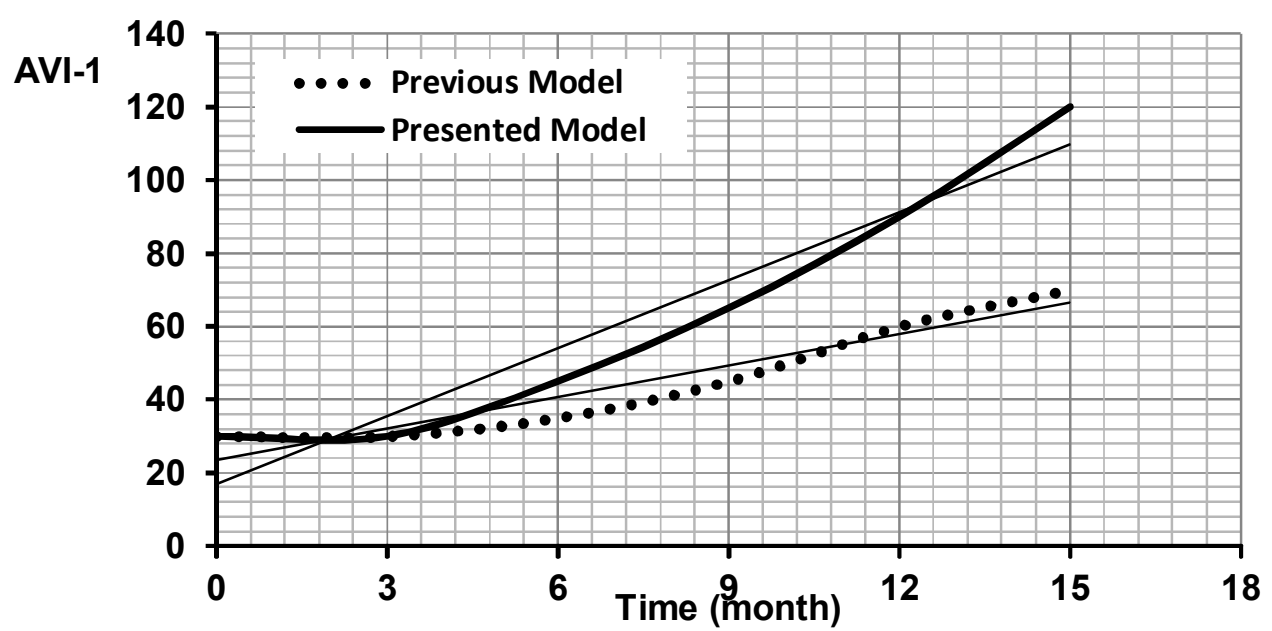

Fig. 4. Comparison between presented and previous KM implementation model for part one of the characteristics in case study (AVI-1)

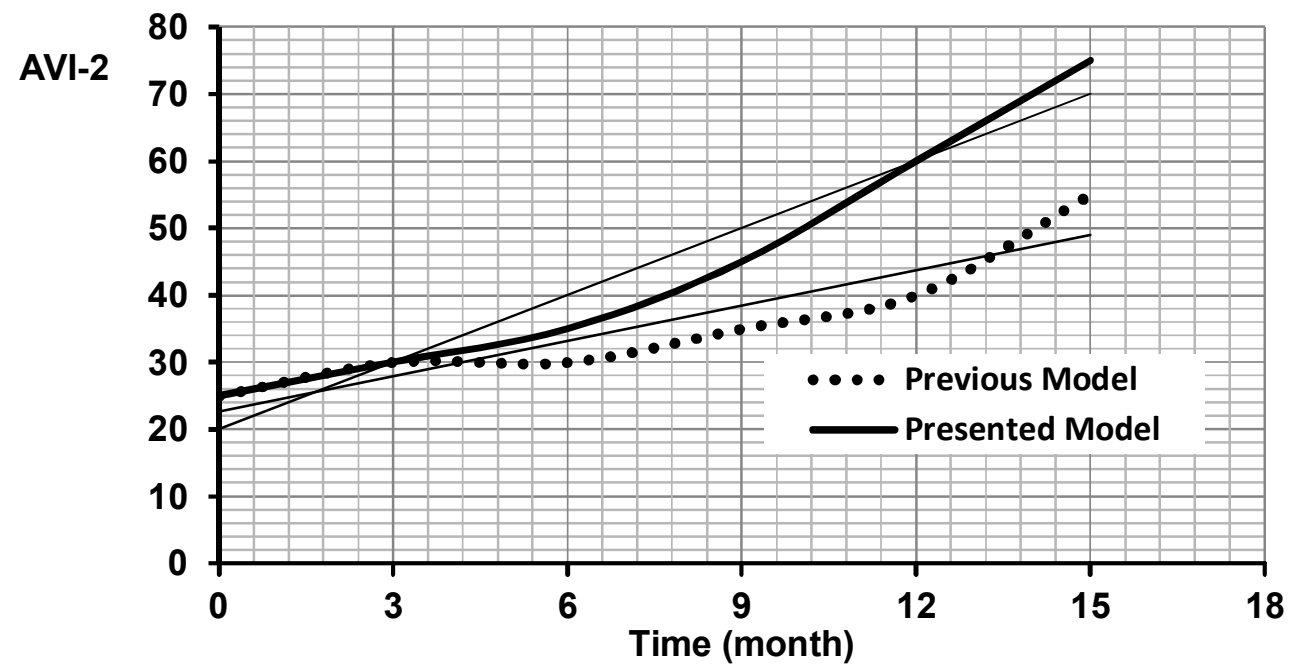

Fig. 5. Comparison between presented and previous KM implementation model for part two of the characteristics in case study (AVI-2)

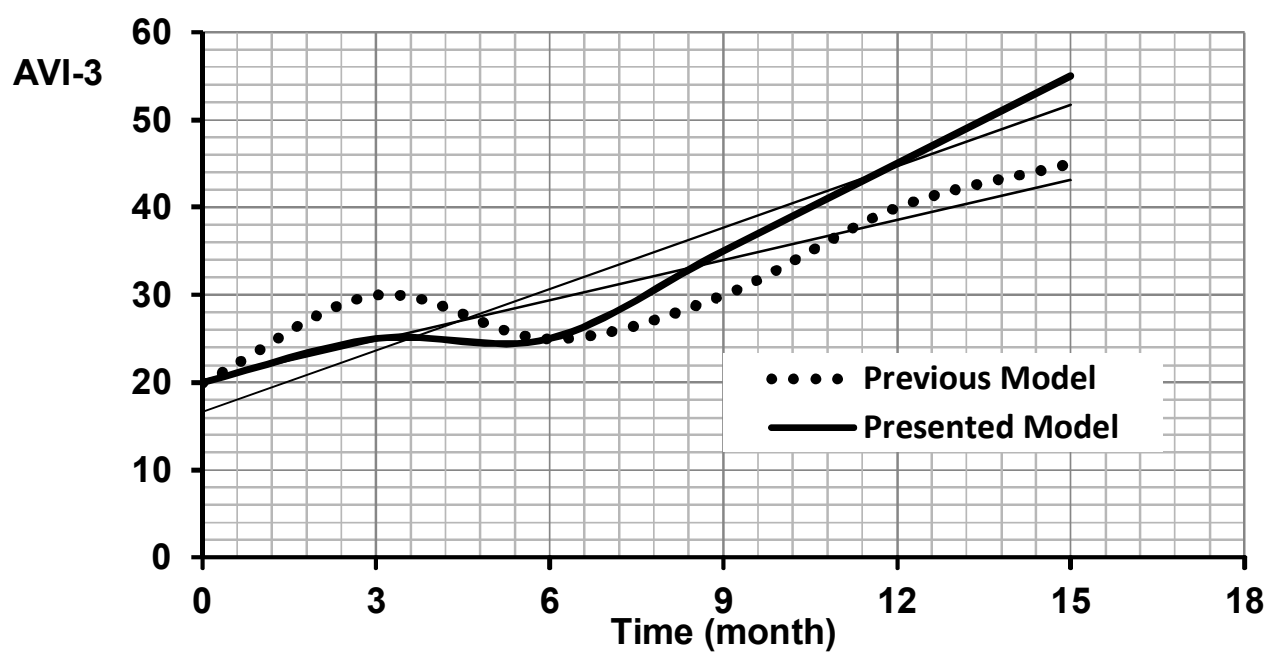

Fig. 6. Comparison between presented and previous KM implementation model for part three of the characteristics in case study (AVI-3) 


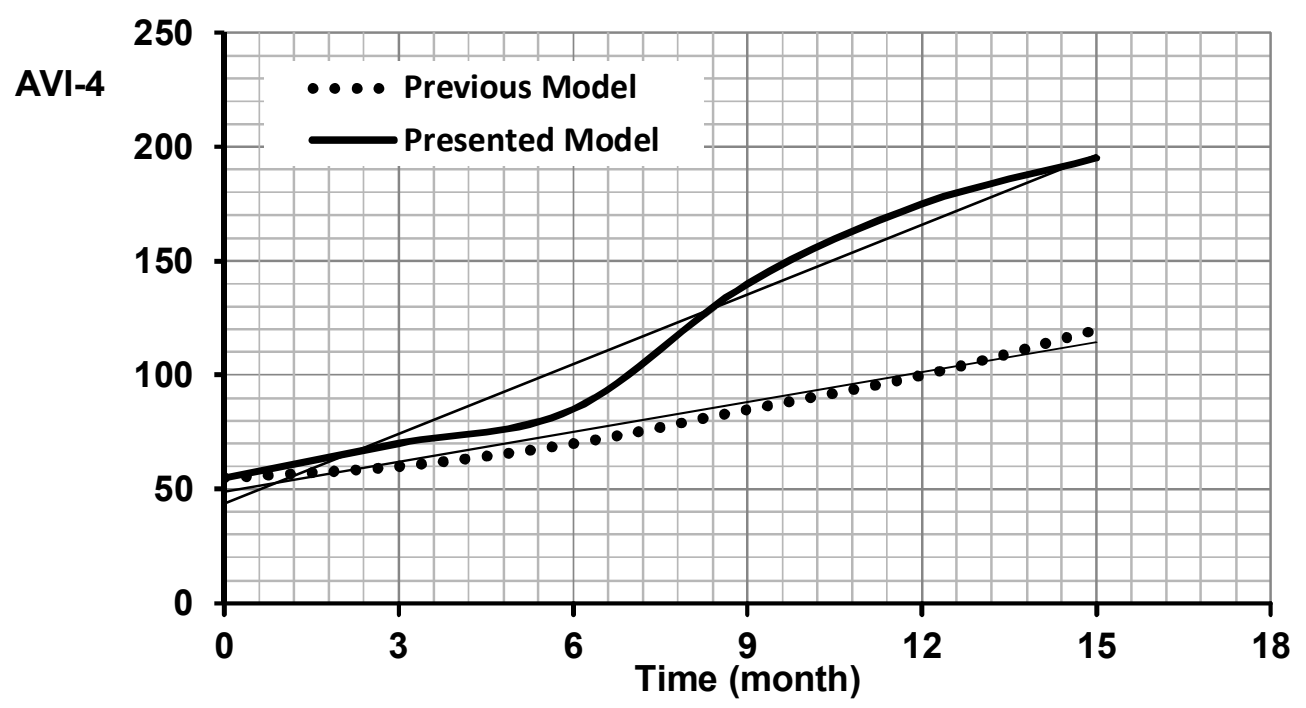

Fig. 7. Comparison between presented and previous KM implementation model for part four of the characteristics in case study (AVI-4)

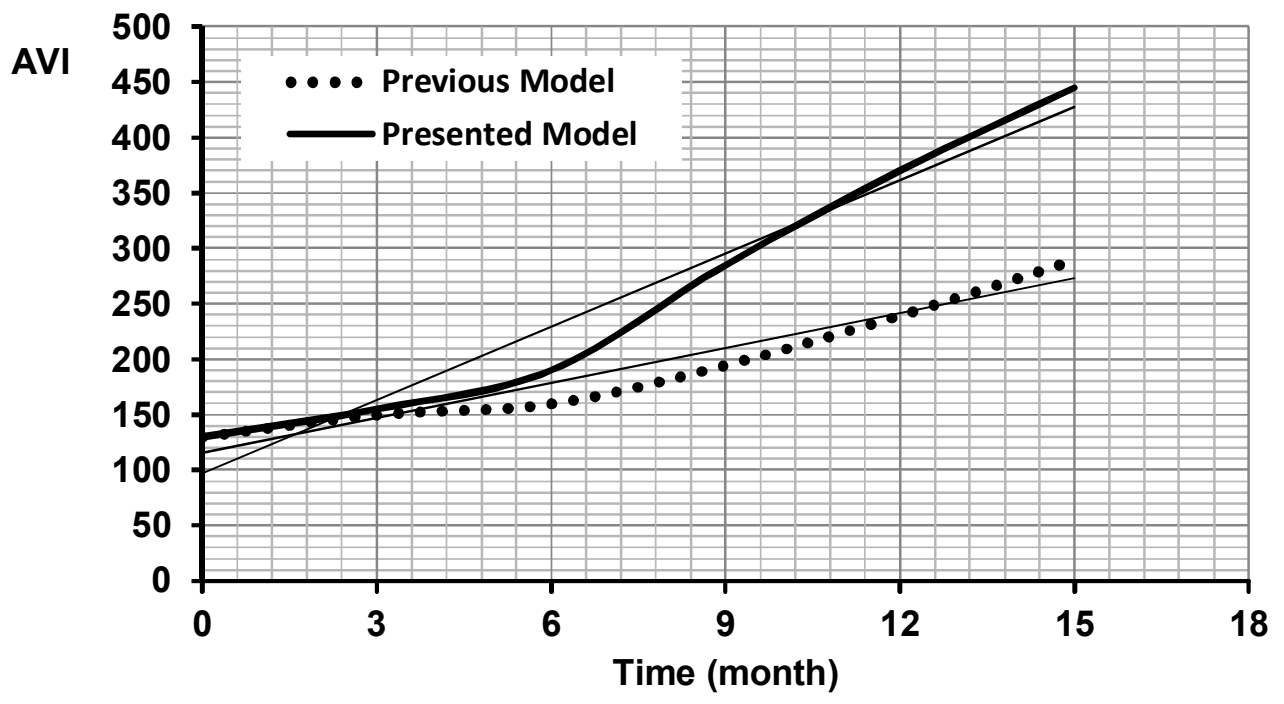

Fig. 8. Comparison between presented and previous KM implementation model for all of the characteristics in case study (AVI)

\section{Conclusions}

A new model is designed and introduced for knowledge management implementation especially for applying in projects related to historic buildings such as restoration. A peer review is carried out on previously presented of KM implementation models which were developed in a general manner without any consideration of an especial area of concern.

Some main improvement potentials in main core components of KM including peoples, processes, and technologies are recognized and the new model developed considering operational processes to cover the improvement potentials. It is concluded that the improvement can be achieved by definition a finger frame pattern for KM implementation model including four main tiers as well as an intelligent communication medium as tier interface. The lower frame of model consists of basic and operator tiers and the top one consists of monitoring and goals tier. Based on details operational 
processes which are designed in each tier, it is concluded that three main components of KM block diagrams consist of knowledge identification, acquisition, and prevention especially for a HBs-related project is appropriately covered in basic tier.

The other three components of inner cycle of KM basic diagram consist of using, development, and distribution are also covered in an interface between operators and basic tiers which together with make lower frame of new presented model named as Finger Frame Model (FFM). The model is in a continuous improving stage considering self-monitoring processes in an individual tier with the same name which is located in the upper frame of FFM with the upper layer named as goal tier in which, the main quantitative goals of KM implementation in the project organization are considered. The conceptual diagrams of the new presented model as well as internal processes of each main tier are also presented and discussed. Based on the overall explanations, some main benefits and risks are discussed for new presented model and outlooks are debated.

The new presented method for knowledge management implementation is applied in a real case for a consulting engineer involved in a restoration project on Ghazagh-Khaneh, a building in the center of Tehran, capital of I.R. of Iran. The results are characterized using 40 items that affect the added value gained from knowledge management implementation in a project organization. The results captured in a 15-month period in one-month intervals and the graphs are investigated. It is concluded that:

1. Maximum improvement in the project organization or maximum added valued achieved from KM model implementation is seen for AVI-1 with about $70 \%$ increasing comparing to previous model. It seems that the easiness in knowledge transferring is majorly achieved in new presented model.

2. Minimum improvement is seen for AVI-3 with less than $25 \%$ increasing. It can be concluded that there is the minimum change in cost-related items in the project organization after implementation of new presented model.

3. Essential time duration about 7-8 months is needed to reflect any changes in the project organization caused by new implementation model.

4. An overall improvement about $50 \%$ in sum of all indexes (AVI) show that the new implementation model introduced here can be assumed as a model that works efficiently in projects organization related to the historic building restoration.

\section{References}

Bade, D. (2011). An Approach to Researching Cultural Heritage Management on Conservation Islands in New Zealand. Graduate Journal of Asia-Pacific Studies, 7(2), 62-77.

Bhatt, A., \& Ganesh C. (2000). A resource-based perspective of developing organizational capabilities for business transformation. Knowledge and Process Management, 9(3), 150-161.

Barley, S. R. (1986). Technology as an occasion for structuring: Evidence from observations of CT scanners and the social order of radiology departments. Administrative Science Quarterly, 31(1), 78-108.

Bowers, J. (1970). Managing the Resource Allocation Process. Englewood Cliffs, NJ: Irwin.

Chesbrough, H. W. (1999). The organizational impact of technological change: a comparative theory of national institutional factors. Industrial and Corporate Change, 8(3), 447-485.

Chutinan S. (2008). Cultural heritage interpretation, management, and promotion: Phimai historical park. PhD Thesis, $205 \mathrm{p}$.

Ferraro, F., Pfeffer, J., \& Sutton, R. I. (2005). Economics language and assumptions: How theories can become self-fulfilling. Academy of Management Review, 30(1), 8-24.

Hambrick, D. C. (1994). What if the Academy actually mattered?. Academy of Management Review, 19(1), 11-16. 
Hoffman, J. J., Hoelscher, M. L., \& Sherif, K. (2005). Social capital, knowledge management, and sustained superior performance. Journal of Knowledge Management, 9(3), 93-100.

Lustri, D., Miura, I., \& Takahashi, S. (2007). Knowledge management model: practical application for competency development. Learning Organization, The,14(2), 186-202.

Nonaka I. (2001). The knowledge creating company. Harvard Business Review, 96-104.

Tarhuni, S. (2012). Knowledge management in historic buildings conservations: a case study of responsible institution in Tripoli, Libya. Research Seminar Report, School of Architecture, Planning, and Landscape, Newcastle University, 1-11. 